\title{
Supermode-noise suppression using a nonlinear Fabry-Pérot filter in a harmonically mode-locked fiber ring laser
}

Cite as: Appl. Phys. Lett. 81, 4520 (2002); https://doi.org/10.1063/1.1528732

Submitted: 08 July 2002 . Accepted: 17 October 2002 . Published Online: 03 December 2002

Donghui Zhao, Yicheng Lai, Xuewen Shu, Lin Zhang, and Ian Bennion

\section{ARTICLES YOU MAY BE INTERESTED IN}

$120 \mathrm{fs}, 4.2 \mathrm{~nJ}$ pulses from an all-normal-dispersion, polarization-maintaining, fiber laser Applied Physics Letters 103, 12111 (2013); https://doi.org/10.1063/1.4821776

Large energy soliton erbium-doped fiber laser with a graphene-polymer composite mode locker

Applied Physics Letters 95, 141103 (2009); https://doi.org/10.1063/1.3244206

Time domain analysis of a rational harmonic mode locked ring fiber laser

Journal of Applied Physics 90, 2143 (2001); https://doi.org/10.1063/1.1390304

Applied Physics Reviews

Now accepting original research 


\title{
Supermode-noise suppression using a nonlinear Fabry-Pérot filter in a harmonically mode-locked fiber ring laser
}

\author{
Donghui Zhao, ${ }^{\text {a) }}$ Yicheng Lai, Xuewen Shu, ${ }^{\text {b) }}$ Lin Zhang, and lan Bennion \\ Photonics Research Group, Aston University, Birmingham B4 7ET, United Kingdom
}

(Received 8 July 2002; accepted 17 October 2002)

\begin{abstract}
A simple efficient method for stabilizing a harmonically mode-locked fiber ring laser is proposed. In this method, a linear optical filter and a nonlinear Fabry-Pérot filter in which the refractive index is optical intensity dependent are located in the laser cavity. The linear filter is used to select a fixed lasing wavelength, and the Fabry-Pérot filter introduces a negative all-optical feedback mechanism that is able to suppress pulse-to-pulse amplitude fluctuations in the laser cavity. The scheme was experimentally demonstrated using a fiber Bragg grating as the linear filter and a laser diode biased below threshold as the nonlinear Fabry-Pérot, and stable harmonically mode-locked pulses with a supermode noise suppression ratio $>55 \mathrm{~dB}$ were obtained. (C) 2002 American Institute of Physics. [DOI: $10.1063 / 1.1528732]$
\end{abstract}

A stable optical short pulse source with a high-repetition rate is a key component for realizing ultrahigh speed optical communication systems. Of the many potential techniques, active mode locking of an erbium-doped fiber laser (EDFL) leads to an attractive component for generating picosecond pulses with $\mathrm{GHz}$ repetition rates in the $1.5 \mu \mathrm{m}$ wavelength region. ${ }^{1,2}$ However, the stability of the active mode-locked EDFL has proven to be a problematic issue that demands a realistic solution before the source can be considered for practical application. Since the overall EDFL cavity is typically long, the output of the mode-locked fiber laser tends to be inherently unstable due to the effects of fluctuations in the signal polarization state and the cavity length caused by mechanical vibrations and temperature variations. In addition, in order to obtain the $\mathrm{GHz}$ repetition rates needed for highspeed communication links, the harmonic mode-locking technique is used: it is a consequence of this technique that many supermodes oscillate simultaneously, competing with each other, and resulting in fluctuations of the output pulse amplitude. This effect represents one of the principal noise sources in the harmonically mode-locked fiber ring laser.

Various methods for reducing the supermode noise in actively mode-locked fiber lasers have been sought and reported. Shan et al. developed a pulsed phase-lock technique to suppress the noise through spatial hole burning. ${ }^{3}$ A fast intensity-dependent gain in the cavity has been exploited to equalize the pulse amplitude and thereby suppress the noise. ${ }^{4,5}$ In Ref. 5 pulse amplitude equalization was achieved through the interplay between intracavity self-phase modulation (SPM) and filtering: in a cavity with anomalous dispersion, higher intensity pulses are spectrally broadened through SPM and, thus, experience higher loss through a fixed filter. However, in order to achieve sufficient SPM, a long cavity is necessitated by the small fiber nonlinearity and limited cavity pulse power. By operating the laser at a lower harmonic using a composite cavity structure, ${ }^{6,7}$ the number of super-

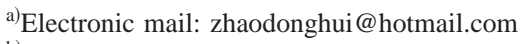

${ }^{\mathrm{b}}$ Present address: Indigo Photonics Ltd., Faraday Wharf, Birmingham B7 4BB, UK. modes can be reduced, but no measurable reduction of the overall supermode noise is obtained. By attaching a subcavity with a free spectral range equal to the modulation frequency, the unwanted supermodes can be suppressed. ${ }^{8}$ The major disadvantage of this method is that it requires interferometric stabilization of the subcavity with respect to the modulation frequency. Another technique that has recently been discussed achieves pulse-intensity limiting through two-photon absorption by a semiconductor mirror. ${ }^{9}$

In this letter, we propose and demonstrate an approach to suppress the supermode noise in an harmonically modelocked fiber laser based on the relatively simple expedient of introducing a combination of a linear optical filter and a nonlinear Fabry-Pérot filter (NFPF) into the laser cavity. We find that the method is easily realized, and provides significant and efficient supermode noise suppression when the lasing wavelength is properly set.

Figure 1 illustrates the experimental arrangement. The gain in laser loop was provided by an erbium-doped fiber amplifier. A fiber Bragg grating (FBG) was used as a linear filter that was responsible for selecting the operating wavelength of the laser. The reflectivity of the FBG used here was

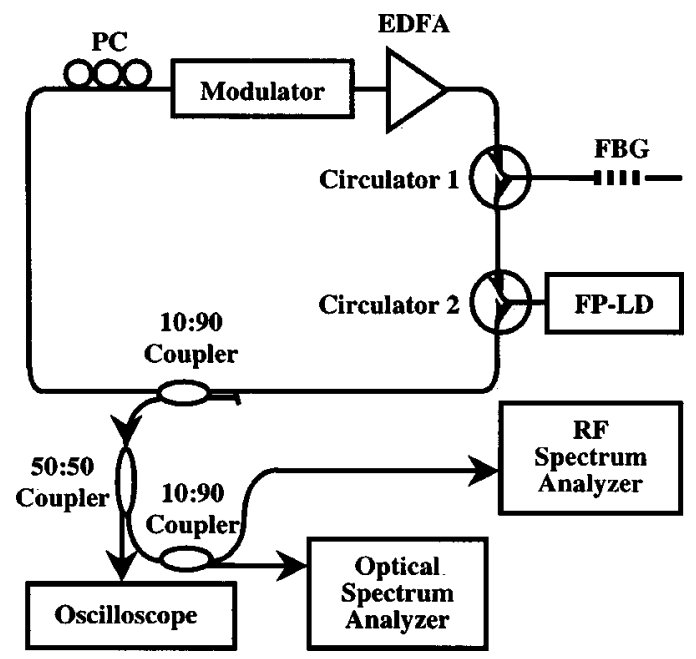

FIG. 1. Schematic diagram of the experimental arrangement. PC: Polarization controller; EDFA: erbium doped fiber amplifier. 


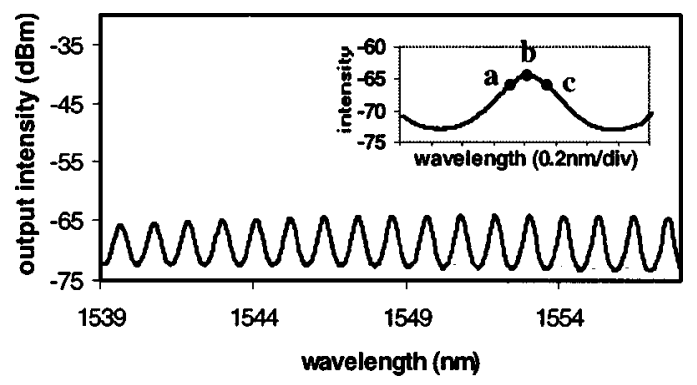

FIG. 2. Optical spectrum of the NFPF with drive current set to $8.3 \mathrm{~mA}$. The inset shows one peak of the NFPF.

about $95 \%$, and its center wavelength and bandwidth were 1548.14 and $0.17 \mathrm{~nm}$, respectively. $\mathrm{A}^{\mathrm{LiNbO}_{3}}$ MachZehnder amplitude modulator was inserted into the cavity to provide gain (or loss) modulation. Because of the polarization sensitivity of the $\mathrm{LiNbO}_{3}$ modulator, a polarization controller was inserted at its input port to optimize the modulation. A commercial, fiber-pigtailed Fabry-Pérot laser diode (FPLD), biased below threshold, was used as the NFPF. The cavity mode spacing and the threshold of the FPLD were $1.125 \mathrm{~nm}$ and $11.5 \mathrm{~mA}$, respectively. Both the grating and the NFPF were introduced into the cavity using optical circulators, which also served as isolators to ensure unidirectional oscillation of the ring cavity. The output light was extracted using a 10:90 optical coupler (1550 nm); output pulses were simultaneously monitored using an optical spectrum analyzer, a fast sampling oscilloscope, and a radio frequency (rf) spectrum analyzer.

The stabilization principle for the mode-locked fiber laser is as follows. The FPLD biased below threshold functions as a NFPF through changes in the refractive index of the semiconductor with changing light intensity due to the carrier depletion effect. ${ }^{10}$ Any increase in the injected light intensity increases the stimulated emission and thereby lowers the carrier density. The reduced carrier density increases the refractive index of the NFPF causing the entire reflection spectrum to shift toward longer wavelengths. A typical NFPF reflection spectrum is shown in Fig. 2. When the laser is operated on the positive slope of the reflection peak, such as at point " $a$ " in the inset of Fig. 2, a long wavelength shift of the reflection spectrum decreases the gain of the NFPF at the lasing wavelength, or increases the cavity loss. An increase in the injected light intensity thus induces an increase in cavity loss and, hence, a decrease in light intensity within the cavity. When the response time of the medium is shorter than the pulse width of the injected pulse train, the resulting negative feedback effectively reduces any amplitude fluctuations in the pulse train and, as a result, the supermode noise in the fiber laser is suppressed. If, on the other hand, the laser is operated on the negative slope of the NFPF peak, such as at point " $c$ " in Fig. 2, positive feedback occurs, so the cavity light intensity builds up and laser noise increases.

To verify the operating principle, we first removed the NFPF from the ring cavity, and measured the fundamental frequency of the laser to be $\sim 3.7 \mathrm{MHz}$. When the laser was mode locked at the 384th harmonic $(f=1.420801 \mathrm{GHz})$, the supermode noise suppression ratio (SNSR) was found to be about $35 \mathrm{~dB}$, which is in agreement with most reports of har-

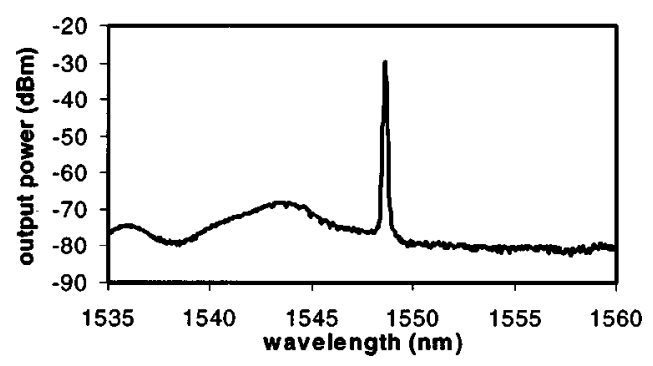

FIG. 3. Typical optical spectrum of the mode-locked fiber ring laser.

monically mode-locked fiber lasers without a stabilization technique applied. ${ }^{3}$

When the NFPF was inserted into the laser ring cavity and dc biased below its threshold at $8.3 \mathrm{~mA}$, the fundamental cavity resonance frequency changed to $\sim 2.66 \mathrm{MHz}$, corresponding to the cavity length of $\sim 75.2 \mathrm{~m}$. The laser was modulated at $1.476341 \mathrm{GHz}$, which corresponds approximately to the 555th harmonic of the ring cavity. Figure 3 shows a typical optical spectrum of the output pulses when the laser was operated at the positive slope side of the NFPF. The center lasing wavelength was $1548.465 \mathrm{~nm}$, effectively determined by the FBG, and the linewidth was about 0.09 $\mathrm{nm}$. Tuning of the laser center wavelength could be readily carried out by applying strain to the grating.

We measured the effectiveness of the noise suppression technique by maintaining all operating conditions constant except for the center wavelength of the FBG, which was sequentially tuned to different positions on the NFPF reflection spectrum. The stability of the laser output was monitored by simultaneously measuring its wave form and $\mathrm{rf}$ spectrum. A sampling oscilloscope was set to infinitepersistence display mode so that every point sampled was displayed and revealed pulse amplitude fluctuations via the breadth of the composite trace.

Figure 4 shows the measured results for the laser operating at three different wavelengths. Figures 4(a1) and 4(a2), respectively, show the wave form and the rf spectrum with
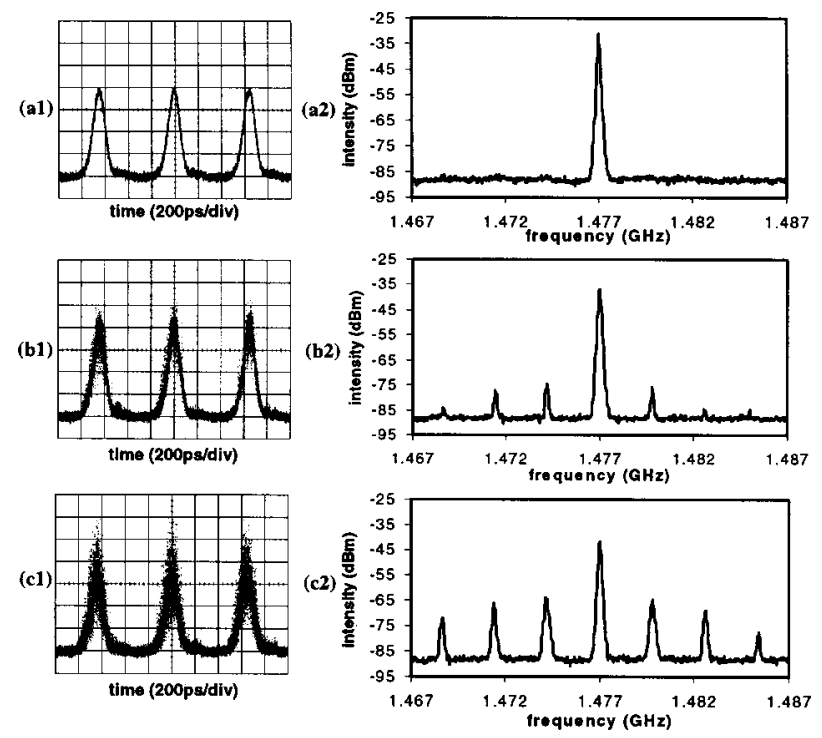

FIG. 4. Wave forms and if spectra of the output pulses when the laser operated at three wavelengths that correspond to points $a, b$ and $c$, respectively, in the inset of Fig. 2. (Span: $40 \mathrm{MHz}$, resolution bandwidth: $100 \mathrm{kHz}$, video bandwidth: $100 \mathrm{kHz}$.) 


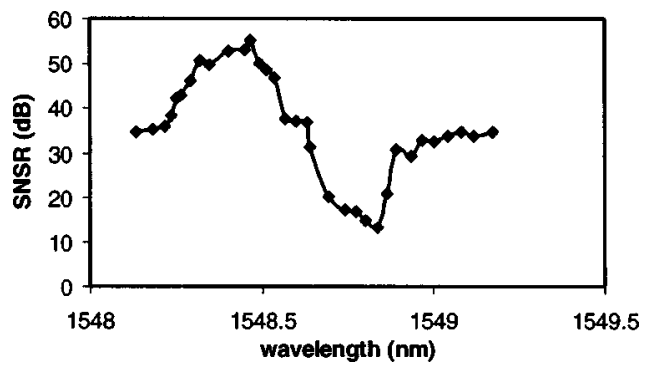

FIG. 5. Supermode noise suppression ratio as a function of the lasing wavelength.

the laser oscillating at $\lambda_{1}=1548.465 \mathrm{~nm}$, corresponding to operation on the positive-slope side of a reflection peak of the NFPF (point $a$ in Fig. 2). It is clear from Fig. 4(a2) that the supermode noise is suppressed by more than $55.2 \mathrm{~dB}$ with respect to the signal: this is a substantial improvement of the $\sim 35 \mathrm{~dB}$ suppression achieved without stabilization. The clear stable pulse train shown in Fig. 4(a1) provides further confirmation of the effectiveness of the technique. With the laser wavelength tuned to $\lambda_{2}=1548.57 \mathrm{~nm}$, corresponding to operation at the highest point of the NFPF reflection peak (point $b$ in the inset of Fig. 2), the results shown in Figs. 4(b1) and 4(b2) were obtained. The appearance of supermodes adjacent to the carrier frequency is clear, and the SNSR was reduced to $37.7 \mathrm{~dB}$, a value similar to that obtained without any stabilization applied. As shown in Fig. 4(b1), the corresponding pulse trace became considerably noisier, indicative of increased amplitude fluctuation of the pulse train. The lack of improvement in this case is to be expected since, at point $b$, the slope of the NFPF reflection characteristic is essentially zero, and the feedback effect is suppressed. When the laser-oscillating wavelength was tuned to the negative-slope side of the NFPF reflection peak, the output exhibited even greater noise than that of the unstabilized laser, due to the onset of the positive feedback effect. Figures 4(c1) and 4(c2) show the results obtained for this case, with the laser operating at $\lambda_{3}=1548.694 \mathrm{~nm}$ : the SNSR degraded to just $22.2 \mathrm{~dB}$, substantially lower than that $(\sim 35 \mathrm{~dB})$ of the unstabilized laser. Note, in Figs. 4(b2) and $4(\mathrm{c} 2)$, there is a sinusoidal-type envelope of supermodes, which is expected to be the result of the low-finesse FP effect due to the FP cavity formed by the polished fiber end surfaces of the adapters.
Figure 5 illustrates the variation of the SNSR value with the wavelength, and shows each of the regimes of operation of the system. In the laser-oscillating wavelength range between 1548.236 and $1548.536 \mathrm{~nm}$, corresponding to operation on the positive side of the reflection characteristic of the NFPF, the SNSR is somewhat higher and the laser stability has substantially improved compared with that of that unstabilized system. The NFPF reflection peak occurs at 1548.57 $\mathrm{nm}$, and at this point there is no significant effect on the SNSR or the stability. Degraded operation with lower stability and SNSR due to the positive feedback effect is apparent in the range of 1548.64-1549.003 $\mathrm{nm}$. With the laser wavelength shorter than $1548.218 \mathrm{~nm}$ or longer than 1449.043 $\mathrm{nm}$, corresponding to the notch in the NFPF characteristic, the laser stability was neither improved nor degraded since, then the feedback effect-whether negative or positive-is very weak. The above repeatable results show that the slope of the NFPF reflection characteristic determines the efficiency of the suppression.

In conclusion, we have demonstrated a simple and effective technique for suppressing the supermode beat noise in an actively mode-locked fiber ring laser. By using a FBG as a linear filter and a laser diode as a nonlinear Fabry-Pérot filter, the stability of the laser is much improved and the SNSR increased to $>55 \mathrm{~dB}$, an improvement of some $20 \mathrm{~dB}$ over the unstabilized value.

This study was carried out with the support of the U.K. Engineering and Physical Sciences Research Council.

${ }^{1}$ T. Pfeiffer and G. Veith, Electron. Lett. 29, 1849 (1993).

${ }^{2}$ T. F. Carruthers and I. N. Duling III, Opt. Lett. 21, 1927 (1996).

${ }^{3}$ X. Shan and D. M. Spirit, Electron. Lett. 29, 979 (1993).

${ }^{4}$ C. R. Doerr, H. A. Haus, E. P. Ippen, M. Shirasaki, and K. Tamura, Opt. Lett. 19, 31 (1994).

${ }^{5}$ M. Nakazawa, K. Tamura, and E. Yoshida, Electron. Lett. 32, 461 (1996).

${ }^{6}$ N. Onodera, Electron. Lett. 33, 962 (1997).

${ }^{7}$ O. Pottiez, O. Deparis, R. Kiyan, M. Haelterman, P. Emplit, P. Megret, and M. Blondel, IEEE J. Quantum Electron. 38, 252 (2002).

${ }^{8}$ G. T. Harvey and L. F. Mollenauer, Opt. Lett. 18, 107 (1993).

${ }^{9}$ E. R. Thoen, M. E. Grein, E. M. Koontz, E. P. Ippen, H. A. Haus, and L. A. Kolodziejski, Opt. Lett. 25, 948 (2000).

${ }^{10} \mathrm{~S}$. Li, K. T. Chan, and C. Lou, IEEE Photonics Technol. Lett. 10, 1094 (1998). 